\title{
Radial distribution of dust properties in nearby galaxies
}

\author{
J.C. Muñoz-Mateos ${ }^{1}$, A. Gil de Paz ${ }^{1}$, S. Boissier ${ }^{2,3}$, J. Zamorano ${ }^{1}$, D.A. Dale ${ }^{4}$, \\ P.G. Pérez-González ${ }^{1}$, J. Gallego ${ }^{1}$, B.F. Madore ${ }^{5}$, G. Bendo ${ }^{6}$, M. Thornley ${ }^{7}$, A. \\ Boselli $^{2,3}$, V. Buat ${ }^{2,3}$, D. Calzetti ${ }^{8}$, and J. Moustakas ${ }^{9}$
}

\begin{abstract}
We present a detailed analysis of the radial distribution of dust properties (extinction, PAH abundance and dust-to-gas ratio) in 57 galaxies in the SINGS sample, performed on a multi-wavelength set of UV, IR and radio surface brightness profiles, combined with published molecular gas profiles and metallicity gradients.
\end{abstract}

\section{Introduction}

Analyzing the properties of interstellar dust is of particular importance for two reasons: dust affects our view of galaxies at different wavelengths, by absorbing UV and optical light and reemitting it in the infrared, and it also constitutes an important element in the chemical evolution of the ISM. As for the first issue, correcting for dust extinction is usually the main source of uncertainty when measuring properties such as the SFR, age or metallicity. By deriving radial attenuation profiles we can recover the intrinsic UV, optical and near-IR profiles, which trace the radial distribution of stars of different ages.

Secondly, dust is also a key ingredient in the chemical enrichment of the ISM. Different species are injected into the ISM at different rates, leading to temporal variations of the relative abundances of the different dust constituents. For instance, the abundance of polycyclic aromatic hydrocarbons (PAHs) relative to other dust

\footnotetext{
${ }^{1}$ Departamento de Astrofísica y CC. de la Atmósfera, Universidad Complutense de Madrid. e-mail: jcmunoz@astrax.fis.ucm.es

${ }^{2}$ Observatoire Astronomique de Marseille-Provence, Laboratoire d'Astrophysique de Marseille.

${ }^{3}$ Centre National de la Recherche Scientifique.

${ }^{4}$ Department of Physics and Astronomy, University of Wyoming, Laramie, WY

${ }^{5}$ Observatories of the Carnegie Institution of Washington, Pasadena, CA

${ }^{6}$ Astrophysics Group, Imperial College, Blackett Laboratory, London

${ }^{7}$ Department of Physics and Astronomy, Bucknell University, Lewisburg, PA

${ }^{8}$ Department of Astronomy, University of Massachusetts, Amherst, MA

${ }^{9}$ Center for Cosmology and Particle Physics,New York University, New york, NY
} 
species is expected to vary with the age of the stellar populations and correlate with the metal abundance of the gas. The dust-to-gas ratio measures the amount of metals that get locked in dust grains, and also depends on metallicity. Therefore, the radial change of these parameters can be used to constrain the timescales in which disk evolution takes place.

\section{Sample, data and procedure}

The SINGS sample ([15]) consists of 75 nearby galaxies selected to cover the range in morphological type, luminosity and FIR/optical luminosity observed in the local universe. All galaxies are closer than $30 \mathrm{Mpc}$, with the median distance being $10 \mathrm{Mpc}$. Eighteen SINGS galaxies were not suitable for our purposes, due to being unresolved and/or undetected in at least one of the MIPS bands. Thus, the final subsample includes 57 galaxies.

Our main data-set consists of GALEX (FUV, NUV), IRAC (3.6, 4.5, 5.8 and $8.0 \mu \mathrm{m})$ and MIPS $(24,70$ and $160 \mu \mathrm{m})$ images, plus HI maps from the THINGS survey ([18]) for 21 of them. All these images were convolved with different kernels [12] in order to match the shapes and resolution of their PSFs to the one of the MIPS $160 \mu \mathrm{m}$ images. Prior to this, foreground stars, background galaxies and artifacts were masked and interpolated over to avoid contamination when degrading the images. We complemented these data with metallicity gradients from Moustakas et al. (2008, in prep) and published CO profiles.

The radial profiles were obtained using the IRAF task ELLIPSE, measuring the mean intensity along elliptical isophotes with a constant increment of 48" (larger than the $160 \mu \mathrm{m}$ PSF FWHM) along the semimajor axis. Both the ellipticity and the position angle were kept fixed, and were set equal to those of the $\mu_{B}=25$ mag $\operatorname{arcsec}^{-2}$ isophote from the RC3 catalog ([5]) and NED.

\section{Extinction profiles}

Several studies (see e.g. [19], [3] and references therein) have shown that the TIRto-FUV ratio is a robust tracer of the internal extinction in star-forming galaxies, in the sense that it depends weakly on details such as the relative geometry of stars and dust, the shape of the extinction curve, or the star formation history (SFH). The dependence of the TIR-to-FUV ratio on the mean age of the stellar populations has been further investigated in [4]. These authors provide a SFH-dependent calibration to estimate the UV attenuation, taking into account the extra dust heating contributed by older stars.

Following these prescriptions, we have derived radial UV-attenuation profiles from the TIR-to-FUV ratio. The overall level of extinction varies along the Hubble sequence, reaching a maximum in $\mathrm{Sb}-\mathrm{Sbc}$ galaxies. On average, in these spirals the 
attenuation ranges from $A_{\mathrm{FUV}} \sim 2.5 \mathrm{mag}$ in the central regions to $\sim 1.5 \mathrm{mag}$ in the outer ones, although with large scatter. The extinction is $\sim 1$ mag lower in earlier spirals, and it goes below $0.5 \mathrm{mag}$ in Sdm spirals and irregulars.

The slope of the UV spectrum $\beta$-or, equivalently, the (FUV-NUV) color- has been proposed as an indirect tracer of dust attenuation in starburst galaxies when FIR data are not available ([13],[17]). The relation is not so tight for normal starforming galaxies ([11]), although using radial profiles seems to decrease the scatter ([1]). Fig. 1 shows that the so-called IRX- $\beta$ relation is shifted towards redder UV colors with respect to the one found in starburst galaxies. Indeed, SFH history seems to be the primary driver for this departure (although maybe not the only one). Annular regions with lower SFR per unit of mass - as traced by the extinction-corrected (FUV $-3.6 \mu \mathrm{m}$ ) color - lie further away from the starburst relation than those regions hosting more active SF activity. This is in agreement with the predictions of theoretical models ([16]), which attribute this behavior to old stellar populations being intrinsically redder in the UV.

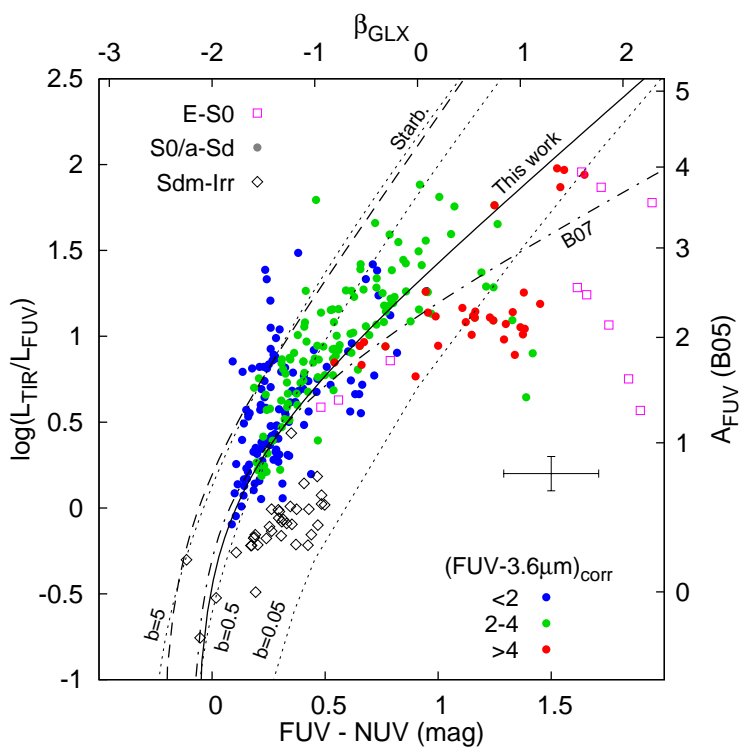

Fig. 1 Ratio of the total infrared to FUV luminosity as a function of the UV spectral slope ( $\beta_{\mathrm{GLX}}$ ) or, equivalently, the UV color, for all the profiles in the sample. The rightmost y-axis shows the internal extinction in the FUV for a given TIR-to-FUV ratio, according to the age-independent fits of [3], only valid for late-type systems (see text). For the disk-like galaxies, a color scheme is used to map the intrinsic (FUV $-3.6 \mu \mathrm{m}$ ) color, corrected for internal extinction using the age-dependent calibration of [4]. The solid line corresponds to a fit to the data-points bluer than (FUV-NUV) $=0.9$, excluding three starburst galaxies. The dashed line shows the relation found by [1] for spiral galaxies using GALEX and IRAS data. The dot-dashed line is the mean relation for the starburst galaxies of [17]. The three dotted lines are model predictions for different values of $b$, the present to pastaveraged SFR ([16]). The mean error bars (including zero-point errors in all bands) are also shown. 


\section{PAH abundance}

We have fitted our radial SEDs with the dust models of Draine \& Li ([6]). As a result, we have derived the radial variation of the PAH abundance $q_{\mathrm{PAH}}$, that is, the fraction of the total dust mass in the form of PAHs. It is known that low-metallicity systems exhibit a paucity of aromatic emission (see e.g. [7]), but the origin of such a trend is still debated. Several authors have invoked an evolutionary origin for this trend ([8] and references therein). PAHs are thought to form in the envelopes around carbonrich AGB stars, being later injected into the ISM through a recycling process that takes place over timescales of a few Gyr. The remaining dust species are believed to condense out of ejecta from supernovae in much shorter timescales. Hence, the different speed of both processes would naturally lead to a progressive increase in $q_{\text {PAH }}$ with time, explaining the observed PAH-metallicity trend. Conversely, other possible explanations rely on destructive mechanisms of PAH particles exposed to hard UV radiation fields.

In Fig. 2 we plot the PAH abundance at each galactocentric distance as a function of the corresponding metallicity at the same radial bin. Previous works relying on integrated photometry usually needed to compare normal spirals with dwarf galaxies in order to detect and quantify the influence of metallicity. Our spatially-resolved analysis reveals interesting trends in a more limited range of oxygen abundances.

There is a general trend of $q_{\text {PAH }}$ to increase with the oxygen abundance for $12+$ $\log (\mathrm{O} / \mathrm{H})<9$. At larger metallicities, however, the slope flattens and even reverses. This is in qualitative agreement with the predictions of the models of [8], who argue that such a change is due to the different carbon content of AGB stars with different masses and lifetimes. Low-mass AGB stars are not as carbon-rich as high-mass ones ([10]). Since the former have longer lifetimes, they contribute later to the chemical enrichment of the ISM. Therefore, the increase of the PAH abundance relative to other dust species slows down and eventually reverses at larger metallicities.

The four galaxies highlighted in gray have low PAH abundances for their metallicities. Indeed, $q_{\mathrm{PAH}}$ and $(\mathrm{O} / \mathrm{H})$ seem to be anticorrelated in these spirals. All of them belong to earlier Hubble types than the remaining galaxies in the plot. If the the evolutionary scenario described above is driving the reversal of the slope seen in the central regions of late-type spirals, the rapidly declining SFHs of early-type galaxies ([9]) could be enhancing this effect in these objects.

Therefore, the analysis above supports the idea that in spatial scales of a few $\mathrm{kpc}$ (that of our azimuthally-averaged profiles) SFH might be the main driver of the observed trends between metallicity and the PAH abundance.

\section{Dust-to-gas ratio}

It is known that the dust-to-gas ratio of star-forming galaxies increases with metallicity (see e.g. [14] and references therein). Such a correlation is expected, since dust grains are formed out of metals in the ISM resulting from the stellar nucleosynthesis. 


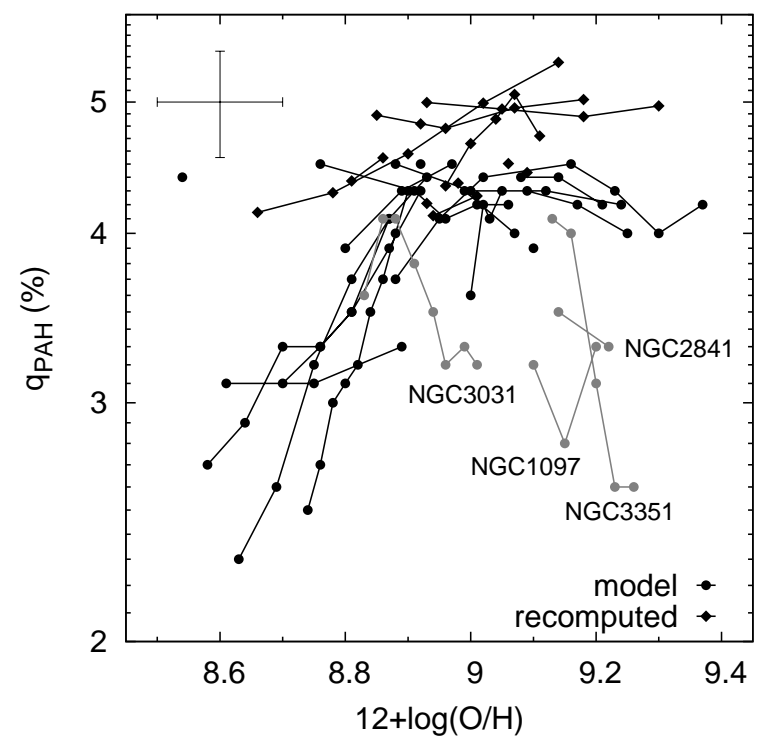

Fig. 2 Fraction of the dust mass contributed by PAHs against the metallicity at each radial bin. Points at different galactocentric distances within the same galaxy are connected by a solid line. Data-points with $q_{\mathrm{PAH}}=4.6 \%$ are 'saturated', meaning that their PAH abundances correspond to the current upper limit of the models. For these points we have recomputed $q_{\text {PAH }}$ from empirical fits derived from the data below that limit (Muñoz-Mateos et al. 2008, in prep.).

In order to analyze this trend as a function of the galactocentric distance, we combined HI data from the THINGS survey with CO profiles compiled from the literature, which were transformed into $\mathrm{H}_{2}$ profiles by means of a metallicity-dependent CO-to- $\mathrm{H}_{2}$ conversion factor ([2]). In Fig. 3 we plot the dust-to-gas ratio against the gas-phase oxygen abundance, for all the SINGS galaxies with available spatiallyresolved metallicities, $\mathrm{HI}$ and $\mathrm{CO}$ data. As a reference, the dashed line corresponds to a simple trend in which the dust-to-gas ratio scales linearly with the oxygen abundance.

The dust-to-gas ratio in early-type spirals seems to roughly follow a simple trend in which the dust-to-gas ratio scales linearly with the oxygen abundance. In late type ones, however, the outer and less metallic regions tend to deviate from that behavior, exhibiting a steeper dependency on metallicity. A number of factors might explain this behavior. Since we lack spatially-resolved submillimeter data, our dust mass surface densities might not be strongly constrained if significant amounts of cold dust $(T<15 \mathrm{~K})$ reside in the disks of these late-type spirals. Conversely, these low dust-to-gas ratios could be due to the presence of large reservoirs of gas that have not still undergone any SF activity, necessary to produce and inject metals into the ISM. 


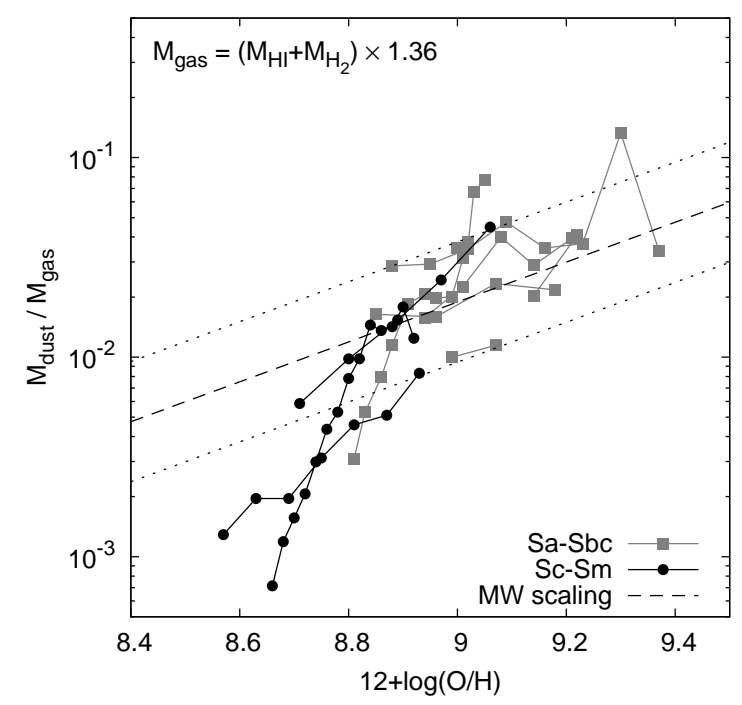

Fig. 3 Dust-to-gas ratio against metallicity at different galactocentric distances. Data-points belonging to the same galaxy have been connected. The dashed line marks a simple scaling law, $M_{\text {dust }} / M_{\text {gas }}=\left(M_{\text {dust }} / M_{\text {gas }}\right)_{\mathrm{MW}} \times(\mathrm{O} / \mathrm{H}) /(\mathrm{O} / \mathrm{H})_{\mathrm{MW}}$, and the dotted lines show variations of a factor of 2 around it.

\section{References}

1. Boissier, S., et al. 2007, ApJS, 173, 524

2. Boselli, A., Lequeux, J., \& Gavazzi, G. 2002, A\&A, 384, 33

3. Buat, V., et al. 2005, ApJ, 619, 51

4. Cortese, L., Boselli, A., Franzetti, P., Decarli, R., Gavazzi, G., Boissier, S., \& Buat, V. 2008, MNRAS, 386, 1157

5. de Vaucouleurs, G., de Vaucouleurs, A., Corwin, H.G., Buta, R.J., Paturel, G., \& Fouqué, P. 1991, Third Reference Catalogue of Bright Galaxies (RC3) (Springer-Verlag)

6. Draine, B. T., \& Li, A. 2007, ApJ, 657, 810 (DL07)

7. Draine, B. T., et al. 2007, ApJ, 663, 866

8. Galliano, F., Dwek, E., \& Chanial, P. 2008, ApJ, 672, 214

9. Gavazzi, G., Bonfanti, C., Sanvito, G., Boselli, A., \& Scodeggio, M. 2002, ApJ, 576, 135

10. García-Lario, P. 2006, in Planetary Nebulae in our Galaxy and Beyond, IAU symp. 234 p.63, eds. M. J. Barlow and R. H. Méndez, Cambridge University Press

11. Gil de Paz, A., et al. 2007, ApJS, 173, 185

12. Gordon, K. D., Engelbracht, C. W., Rieke, G. H., Misselt, K. A., Smith, J. D. T., \& Kennicutt, R. C., Jr. 2008, ApJ, 682, 336

13. Heckman, T. M. et al. 1995, ApJ, 452, 549

14. Hirashita, H., Tajiri, Y. Y., \& Kamaya, H. 2002, A\&A, 388, 439

15. Kennicutt, R. C., Jr., et al. 2003, PASP, 115, 928

16. Kong, X., Charlot, S., Brinchmann, J., \& Fall, S. M. 2004, MNRAS, 349, 769

17. Meurer, G. R., Heckman, T. M., Calzetti, D. 1999, ApJ, 521, 64

18. Walter, F., et al. 2008, ApJ, submitted

19. Witt, A.N., \& Gordon, K.D. 2000, ApJ, 528, 799 